\title{
Endogenous sex hormones and cardiovascular disease in postmenopausal women: new but conflicting data
}

\author{
Pierre-Yves Scarabin \\ INSERM U1018, Center for Research in Epidemiology and Population Health, France and Paris-Sud University, Villejuif, France \\ Correspondence to: Pierre-Yves Scarabin. INSERM U1018, Center for Research in Epidemiology and Population Health, France and Paris-Sud \\ University, Villejuif, France. Email: pierre-yves.scarabin@inserm.fr. \\ Comment on: Zhao D, Guallar E, Ouyang P, et al. Endogenous Sex Hormones and Incident Cardiovascular Disease in Post-Menopausal Women. J \\ Am Coll Cardiol 2018;71:2555-66.
}

Submitted Oct 19, 2018. Accepted for publication Nov 06, 2018.

doi: $10.21037 / \mathrm{atm} .2018 .11 .18$

View this article at: http://dx.doi.org/10.21037/atm.2018.11.18

Cardiovascular disease is the leading contributor to morbidity and mortality in women. The relative delay in the onset of coronary heart disease (CHD) in women compared with men has been attributed largely to the putative protective effects of estrogens before menopause. However, epidemiological data have often been misinterpreted and, although CHD incidence is higher in postmenopausal compared with premenopausal women, the relative contribution of ageing and menopause itself remains uncertain. First, CHD incidence increases exponentially with age in women but this dramatic rise is observed in both genders and CHD mortality rates do not accelerate at or after natural menopause (1). Thus, there is no rebound in CHD risk among women around age 50 years as often claimed. Second, there is no convincing causeeffect relationship between natural menopausal status and CHD (2). While women who experience early menopause have increased CHD risk (3), there is evidence that cardiovascular health may determine the timing of menopause rather the reverse (4). There may be shared risk factors, such as smoking, that result in premature menopause and increase the risk of adverse health outcomes (5). Similarly, women who underwent bilateral oophorectomy are at higher risk of cardiovascular and all-cause mortality (2) but directionality of this association cannot be addressed by observational studies and reproductive health may be only a marker of subsequent cardiovascular disease (6).

In this context, determining the contribution of endogenous sex hormones to the development of cardiovascular disease among postmenopausal women seems particularly relevant. Despite extensive experimental research on the cardiovascular effects of estrogens, very few studies have so far examined the association between circulating sex hormone levels and cardiovascular outcomes. In a recent issue of the fournal of the American College of Cardiology (7), Zhao et al. investigate the relationship of endogenous sex hormone levels with incident cardiovascular disease, CHD and heart failure among 2,834 postmenopausal women participating in the Multi-Ethnic Study of Atherosclerosis (MESA). During 12 years of follow-up, 283 cardiovascular disease events including 171 CHD occurred. In multivariable-adjusted models, higher estradiol levels were associated with a lower CHD risk, whereas higher levels of testosterone were associated with an elevated CHD risk. A higher testosterone/estradiol ratio was associated with an increased incidence of CHD and heart failure events. No association of sex hormones with the risk of stroke was observed.

Notable strengths of the study include a well-designed, multiethnic cohort based, population study, a long duration of follow-up and an adequate number of incident CHD events. Importantly, exclusion of women using hormone therapy (HT) make little change to the results and similar findings were observed when analysis was restricted to well-validated hard events. However, some limitations raise concerns about the reliability of the results. Primary analysis of data included seven hormonal variables combined with five clinical outcomes within three statistical adjustment models. In order to minimize the greatly 
increased probability of declaring false significances, the authors could have addressed the multiple testing problem by adjusting the $\mathrm{P}$ values from a family of hypothesis tests. As emphasized by the authors themselves, some associations may be to the chance. On the other hand, fully adjusted model includes highly inter-correlated biological markers and the negative association between estradiol levels and CHD risk was significant only after simultaneous adjustment for plasma C-reactive protein, fibrinogen, IL-6 and D-Dimer. Overadjustment for these intermediate variables is questionable and may lead to spurious associations.

Another limitation in the MESA study is that serum estradiol and testosterone concentrations were measured using direct radioimmunoassays (RIA), which are less reliable and accurate than chromatography-tandem mass spectrometry, the gold standard, especially at low levels of estradiol among postmenopausal women (8). Nevertheless, the lack of specificity and measurement error of RIA may lead to underestimate but not cause associations between endogenous sex hormones clinical outcomes. Interestingly, in a pooled analysis of 18 prospective studies, stratified by assay type, all measures of serum estradiol including RIA were strongly positively associated with breast cancer risk, without significant heterogeneity between the different assay methods (9).

Are the MESA results consistent with previous findings? The few prospective studies linking endogenous sex hormones and cardiovascular events among postmenopausal women have been negative or contradictory. Conflicting results regarding testosterone and CHD have been reported (10-12) and testosterone concentrations were not associated with the risk of stroke in a recent meta-analysis (13). With respect to estradiol, three prior studies found no significant association with CHD $(10,11,14)$. To my knowledge, the MESA study is the first one reporting a negative association of circulating estradiol with CHD in postmenopausal women. However, this finding contradicts the results of two previous studies that the authors fail to mention $(15,16)$. First, in the Three-City prospective study, high levels of endogenous estradiol have emerged as a new independent predictor of the risk of CHD and stroke among postmenopausal women older than 65 years (15). Second, among postmenopausal women with carotid atherosclerosis who participated to the Rotterdam Study, high levels of circulating estradiol were associated with both increased likelihood of having unstable carotid plaque and increased risk of stroke (16). While amalgamating the findings from postmenopausal HT and endogenous estrogens may be not appropriate, the results of these two cohort studies reinforce the timing hypothesis which assumes harmful effects of estrogens in the elderly among women with underlying atherosclerosis in contrast to protective vascular effects in healthy younger women (17). The results of the MESA study suggesting beneficial effects of estrogens on vascular system among women closer to menopause may also be in line with the timing hypothesis. The age of women enrolled in the cohorts as well as their level of CHD risk factor may therefore be a critical factor to explain the discrepancies between studies.

Mechanistic interpretation of current data on sex steroid and CHD is complex. The vascular effects of estrogens are best documented and extensive experimental data have shown major atheroprotective action of oestradiol (18). These beneficial effects include vasorelaxation via endothelial NO and prostacyclin production, and favorable lipoprotein profile. However, other data suggest deleterious effects of estrogens. Among postmenopausal women, endogenous estradiol levels are positively not negatively related to established CHD risk factors including obesity, dyslipidemia, diabetes and C-reactive protein $(7,15)$. In obese women, both excess fat tissue and high estradiol levels can result in a low-grade inflammation state that can be a mechanism for mediating increased susceptibility to CHD harm of estrogens. One can also speculate that switch from protective to harmful estradiol effect is related to age-related hyper-inflammatory state. On the other hand, high estradiol levels in postmenopausal women have been associated with a procoagulant profile $(19,20)$ that may also represent a central mechanism in the increased CHD risk in postmenopausal women (21). Genetic factors, including estrogen receptor polymorphisms, may also modulate the effects of estrogens on ischemic heart disease (22). A better understanding of the mediators of estradiol effects is still needed and further mechanisms remain to be determined.

Inconsistencies and gaps are particularly obvious in studies of sex hormones among postmenopausal women. Conflicting results and impeding meaningful interpretation make puzzling the etiological role of estrogens and testosterone in cardiovascular disease. The modulating role of sex hormones may differ depending on the type of vascular outcomes. In addition, whether plasma concentrations of sex steroids reflect the entire hormones metabolism at the tissue level remains unclear. Furthermore, HT findings are often used to insight the role of sex steroids but these pharmacologic effects are not necessary relevant 
to endogenous sex hormones and vice versa. For example, oral but not transdermal estrogens result in a hepatic first-pass effect that is responsible for non-physiological changes in sex steroids and impaired protein synthesis. Resulting changes in hemostatic variables may induce blood coagulation activation and increase thrombotic risk among oral estrogens users (23).

Given the rapid growth and improved survival of the aging population, changes in CHD risk in women throughout the long postmenopausal life may have large clinical implications. Overall, while current data reassure about the vascular safety of estrogens in healthy young postmenopausal women, they highlight their potential harm in older women. Future epidemiological research using the most accurate assays will help to determine if and how endogenous sex hormones are related to vascular outcomes and if they can be used to stratify postmenopausal women as high or low risk for cardiovascular disease. Such future data may have the potential to improve preventive strategies.

\section{Acknowledgements}

None.

\section{Footnote}

Conflicts of Interest: The author has no conflicts of interest to declare.

\section{References}

1. Tunstall-Pedoe H. Myth and paradox of coronary risk and the menopause. Lancet 1998;351:1425-7.

2. Atsma F, Bartelink ML, Grobbee DE, et al. Postmenopausal status and early menopause as independent risk factors for cardiovascular disease: a metaanalysis. Menopause 2006;13:265-79.

3. Muka T, Oliver-Williams C, Kunutsor S, et al. Association of Age at Onset of Menopause and Time Since Onset of Menopause With Cardiovascular Outcomes, Intermediate Vascular Traits, and All-Cause Mortality: A Systematic Review and Meta-analysis. JAMA Cardiol 2016;1:767-76.

4. Kok HS, van Asselt KM, van der Schouw YT, et al. Heart disease risk determines menopausal age rather than the reverse. J Am Coll Cardiol 2006;47:1976-83.

5. Hu FB, Grodstein F, Hennekens CH, et al. Age at natural menopause and risk of cardiovascular disease. Arch Intern Med 1999;159:1061-6.
6. Manson JE, Woodruff TK. Reproductive Health as a Marker of Subsequent Cardiovascular Disease: The Role of Estrogen. JAMA Cardiol 2016;1:776-7.

7. Zhao D, Guallar E, Ouyang P, et al. Endogenous Sex Hormones and Incident Cardiovascular Disease in PostMenopausal Women. J Am Coll Cardiol 2018;71:2555-66.

8. Rosner W, Hankinson SE, Sluss PM, et al. Challenges to the measurement of estradiol: an endocrine society position statement. J Clin Endocrinol Metab 2013;98:1376-87.

9. Key TJ, Appleby PN, Reeves GK, et al. Steroid hormone measurements from different types of assays in relation to body mass index and breast cancer risk in postmenopausal women: Reanalysis of eighteen prospective studies. Steroids 2015;99:49-55.

10. Barrett-Connor E, Goodman-Gruen D. Prospective study of endogenous sex hormones and fatal cardiovascular disease in postmenopausal women. BMJ 1995;311:1193-6.

11. Rexrode KM, Manson JE, Lee IM, et al. Sex hormone levels and risk of cardiovascular events in postmenopausal women. Circulation 2003;108:1688-93.

12. Sievers C, Klotsche J, Pieper L, et al. Low testosterone levels predict all-cause mortality and cardiovascular events in women: a prospective cohort study in German primary care patients. Eur J Endocrinol 2010;163:699-708.

13. Holmegard HN, Nordestgaard BG, Jensen GB, et al. Sex Hormones and Ischemic Stroke: A Prospective Cohort Study and Meta-Analyses. J Clin Endocrinol Metab 2016;101:69-78.

14. Chen Y, Zeleniuch-Jacquotte A, Arslan AA, et al. Endogenous hormones and coronary heart disease in postmenopausal women. Atherosclerosis 2011;216:414-9.

15. Scarabin-Carré V, Canonico M, Brailly-Tabard S, et al. High level of plasma estradiol as a new predictor of ischemic arterial disease in older postmenopausal women: the three-city cohort study. J Am Heart Assoc 2012;1:e01388.

16. Glisic M, Mujaj B, Rueda-Ochoa OL, et al. Associations of Endogenous Estradiol and Testosterone Levels With Plaque Composition and Risk of Stroke in Subjects With Carotid Atherosclerosis. Circ Res 2018;122:97-105.

17. Rossouw JE, Prentice RL, Manson JE, et al. Postmenopausal hormone therapy and risk of cardiovascular disease by age and years since menopause. JAMA 2007;297:1465-77.

18. Arnal JF, Douin-Echinard V, Brouchet L, et al. Understanding the oestrogen action in experimental and clinical atherosclerosis. Fundam Clin Pharmacol 2006;20:539-48. 
19. Canonico M, Brailly-Tabard S, Gaussem P, et al. Endogenous oestradiol as a positive correlate of plasma fibrinogen among older postmenopausal women: a population-based study (the Three-City cohort study). Clin Endocrinol (Oxf) 2012;77:905-10.

20. Harrington LB, Marck BT, Wiggins KL, et al. Crosssectional association of endogenous steroid hormone, sex hormone-binding globulin, and precursor steroid levels with hemostatic factor levels in postmenopausal women. J Thromb Haemost 2017;15:80-90.

21. Carcaillon L, Alhenc-Gelas M, Bejot Y, et al. Increased thrombin generation is associated with acute ischemic

Cite this article as: Scarabin PY. Endogenous sex hormones and cardiovascular disease in postmenopausal women: new but conflicting data. Ann Transl Med 2018;6(23):448. doi: 10.21037/ atm.2018.11.18 stroke but not with coronary heart disease in the elderly: the Three-City cohort study. Arterioscler Thromb Vasc Biol 2011;31:1445-51.

22. Scarabin-Carré V, Brailly-Tabard S, Ancelin ML, et al. Plasma estrogen levels, estrogen receptor gene variation, and ischemic arterial disease in postmenopausal women: the three-city prospective cohort study. J Clin Endocrinol Metab 2014;99:E1539-46.

23. Scarabin PY, Oger E, Plu-Bureau G, et al. Differential association of oral and transdermal oestrogen-replacement therapy with venous thromboembolism risk. Lancet 2003;362:428-32. 